\title{
Focal therapy for kidney and prostate cancer
}

\author{
Alexander Kutikov, David A. Kunkle, and Robert G. Uzzo \\ Department of Urologic Oncology, Fox Chase Cancer Center, Philadelphia, Pennsylvania, USA
}

\section{Abstract}

Purpose of review-Surgical excision remains the standard of care for treatment of localized small renal masses (SRMs). Laparoscopic and percutaneous minimally invasive ablative technologies are being increasingly employed in current urologic practice. We review recent literature regarding focal ablative treatments of SRMs.

Recent findings-Most cryoablations are performed using a laparoscopic approach, whereas radiofrequency ablation (RFA) of the SRM is more commonly administered percutaneously. Pretreatment biopsy is performed more often for lesions treated by cryoablation than RFA with a significantly higher rate of indeterminant or unknown pathology for SRMs undergoing RFA versus cryoablation $(P<0.0001)$. Currently available data suggest that cryoablation results in lower retreatments $(P<0.0001)$, less local tumor progressions $(P<0.0001)$ and may be associated with a decreased risk of metastatic progression compared with RFA. It is unclear whether these differences are a function of the technologies or their application. Given the excellent results reported for active surveillance of the SRM in selected patients, the extent to which focal ablation alters the natural history of SRMs has not yet been established.

Summary-Currently, data on the ability of interventions for SRMs to affect the natural history of these masses is lacking. Prospective randomized evaluations of available clinical approaches to SRMs are needed.

\section{Keywords}

active surveillance; cryoablation; partial nephrectomy; radiofrequency ablation; renal cell carcinoma

\section{Introduction}

Excision of localized renal cell carcinoma (RCC) is the current standard of care. Surgical series for small renal masses (SRMs) demonstrate excellent outcomes with 5-year survival approaching 97\% [1]. Minimally invasive ablative technologies such as cryotherapy and radiofrequency ablation have entered the therapeutic arena with similar short and intermediate oncologic results [2]. Recently, data on active surveillance of SRMs have also emerged and demonstrate equivalent CSS rates to ablation in selected patient populations [3]. Here, we present an analysis of the currently available data specifically evaluating focal ablative therapies for the SRM and review these data in the context of natural history (active surveillance) data in an effort to guide clinicians who manage incidental SRMs.

\section{Overview}

More than 54,000 individuals in the United States will be diagnosed with renal malignancy in 2008 and over 13,000 (24\%) will die from the disease, making RCC the most lethal of all

Correspondence to Robert G. Uzzo, MD, Chairman (acting) Department of Surgery, Co-Leader Kidney Cancer Keystone Program, Fox Chase Cancer Center, 333 Cottman Avenue, Philadelphia, PA 19111, USA Fax: +1 215214 1734. e-mail: robert.uzzo@ @fcc.edu. 
genitourinary cancers [4]. Incidental discovery of renal malignancy has been on the rise for nearly two decades [5]. As more localized RCC is diagnosed, the rate of renal surgery has also increased [6,7]. Nevertheless, despite early detection and aggressive treatment approaches, death rates from RCC continue an upward trend. When all-cause mortality of patients diagnosed with RCC is examined in the United States, there is a rise from 1.5 deaths per 100,000 individuals in 1983 to 6.5 in 2002 [6]. These data suggest that a significant portion of localized RCC may be over-treated because early and effective treatments have not impacted RCCrelated mortality. As such, it appears that renal masses differ in their inherent biology, and not all lesions are destined to progress, metastasize and threaten the life of the host.

Surgical resection, especially in young healthy patients with localized lesions continues to be standard management because these individuals have a long life expectancy and salvage therapies for advanced RCC are noncurative [8]. Indeed, surgical series have demonstrated remarkable 5-year cancer-specific survival rates in excess of 95\% $[1,9,10]$. However, elderly patients with RCC present the urologist a unique set of challenges because comorbidities of these individuals compete with RCC for life expectancy. Minimally invasive ablative techniques such as cryoablation and radiofrequency ablation provide a less invasive, less morbid treatment option. Treatment patterns for anatomically uncomplicated SRMs have preferentially shifted toward these ablative technologies at some institutions [11•]. Renal tumor ablation can be performed via an open, laparoscopic or percutaneous route using either cryo or radiofrequency ablative approach. Data have emerged, which suggest that these technologies may have equivalent short and intermediate term outcomes to surgical excision with less morbidity. What remains unanswered is whether focal ablation actually impacts the natural history of the SRM or whether the excellent metastasis-free survival of patients undergoing these treatments is simply a function of the indolent nature of their tumors. In recent years, data on the growth kinetics and metastatic potential of the SRM under active surveillance in selected patients have emerged [3]. Here, we review recent data examining the effects of ablative technologies for the SRM and utilize the active surveillance literature to provide a context for the interpretation of these data.

\section{Cryoablation}

Cryoablation generates tumor destruction by controlled freezing of tissues [2,12]. A probe is placed under direct vision or image guidance into the renal mass and cooling is achieved using liquid argon or nitrogen. The ice ball that results produces protein denaturation and destroys cellular membranes [12]. Furthermore, disruption of the microvasculature sustains cell death due to local tissue ischemia [12]. Temperatures less than $-20^{\circ} \mathrm{C}$ are required for appropriate treatment, and such conditions are experimentally observed $3.1 \mathrm{~mm}$ inside the boundary of the cryotherapy ice ball [13]. Hence, in clinical practice tissues are generally cooled to $-40^{\circ} \mathrm{C}$, and the ice ball is propagated $1 \mathrm{~cm}$ beyond the tumor's edge [2]. Thermocouple or ultrasound can be used to monitor progress of the cryotherapy procedure, and two freeze-thaw cycles appear to achieve the best tissue ablation $[2,14]$. A recent report demonstrated excellent correlation between postcryo radiographic findings and subsequent percutaneous biopsy of treated lesions. Weight et al. [15・•] demonstrated that no lesion that failed to enhance on posttreatment imaging revealed evidence of viable tumor. Although cryotherapy probes can be placed via open exploration, in practice laparoscopic and percutaneous techniques are overwhelmingly used $[16,17]$. Objective comparisons between the laparoscopic and percutaneous approaches are only recently emerging. Finley et al. [18•] reported on 18 patients (19 masses) who underwent percutaneous cryoablation and 19 individuals ( 24 masses) who had laparoscopic cryosurgery at the University of California, Irvine. Mean tumor size was $2.9 \mathrm{~cm}$ (range 1.1-5.4). Procedure times, narcotic requirements, and hospital stays were significantly greater with the laparoscopic approach. In another recent comparison between percutaneous and laparoscopic cryoablation, Bandi et al. [19•] also demonstrated earlier convalescence for the percutaneous approach, but 
did not find a difference in postprocedure opiod requirement nor patient satisfaction. Similarly, Badwan et al. [20] reported a comparative cost analysis of laparoscopic versus percutaneous cryoablation demonstrating that the percutaneous approach (median \$6861) was considerably more cost effective than laparoscopic cryoablation (median \$29,617). Nevertheless, long-term cancer control is yet to be proven equivalent between the percutaneous versus laparoscopic approach. Indeed, some authors believe that percutaneous approach is associated with higher tumor recurrence rates compared with laparoscopic cryoablation [21]. Finally, some anterior tumors are not amenable to a percutaneous approach; although, in clinical practice, this is rather uncommon $[18 \bullet]$.

\section{Radiofrequency ablation}

Radiofrequency ablation (RFA) harnesses a high-frequency alternating current to produce rapid heating of tissues. Denaturation of proteins and destruction of cell membranes results when the tissue temperature rises above $50^{\circ} \mathrm{C}$. Temperature elevation above $105^{\circ} \mathrm{C}$ is avoided because resulting vaporization renders ablation nonuniform and ineffective. Clinically, temperatures between 50 and $100^{\circ} \mathrm{C}$ are employed [22]. Stern et al. [23] recently reported that at intermediate follow-up (mean 30 months) RFA was oncologically comparable with partial nephrectomy for T1a lesions with a disease-specific survival of 93.4\%. A multiinstitutional long-term (at least 40 months) follow-up data also was recently published. Levinson et al. [24.•] describe a $90.3 \%$ long-term recurrence-free survival in 31 individuals with solitary kidney who underwent RFA for SRM. In these and in most other studies, tumor recurrence was defined as a lack of radiographic contrast enhancement and absence of tumor growth $[23,24 \cdot \bullet]$. Recent concerns regarding reliability of imaging to assess RFA efficacy have been raised in the literature. For instance, Weight et al. [15••] reported that over $45 \%$ of renal tumors that fail to show enhancement following RFA, demonstrate viable tumor at a 6-month posttreatment biopsy. Nevertheless, others contend that these findings are explained by the fact that RFA heat fixation preserves tumor architecture and that a biopsy at 6 months is unreliable [25]. Indeed, a recent report by these authors suggests that lack of radiographic enhancement is in fact a reliable marker of cell-kill when a biopsy of RFA-treated masses is performed after 1 year. Raman et al. [26••] describe at least 4 biopsies per lesion no sooner than 1 year following treatment of 20 tumors that did not enhance on cross-sectional imaging following RFA. None of these lesion demonstrated viable tumor remnants on posttreatment biopsies. The authors conclude that a biopsy before 1 year following RFA is unreliable. Despite this data, a recent meta-analysis of available data indicates that RFA may have oncological outcomes, which are inferior to cryoablation [27].

\section{Comparison of cryoablation and radiofrequency ablation}

We recently reported the results of a meta-analysis of the available literature comparing RFA with cryoablation [27]. Case reports, studies that included patients with hereditary or metastatic RCC, and publications that did not include outcome data were all excluded. We analyzed 46 studies from 44 institutions that characterized 1234 localized renal lesions, treated with either open, laparoscopic, or percutaneous cryoablation or RFA. When compared with lesions treated with cryoablation, lesions treated with RFA statistically did not differ in size ( 2.53 versus $2.67 \mathrm{~cm}$ ), patient age (64.5 versus 66.0 years), or follow-up interval (19.3 versus 15.5 months). Pretreatment biopsy was performed more often for lesions treated by cryoablation (82.3\%) than RFA $(62.2 \%)(P<0.0001)$. Moreover, there was a significantly higher rate of indeterminant or unknown pathology for SRMs undergoing RFA (40.4\%) versus cryoablation (24.5\%) $(P<0.0001)$. Repeat ablation was performed more often following RFA $(8.5 \%)$ than cryoablation $(1.3 \%)(P<0.0001)$. 
The meta-analysis revealed that lesions treated with RFA exhibited a highly significant higher rate of local tumor progression than those lesions that were treated with cryoablation ( 4.7 versus $12.3 \%, P<0.0001)$. For both cryoablation and RFA, local tumor progression was defined as radiographic or pathologic evidence of residual disease following initial treatment, regardless of time to recurrence in accordance with the recommendations of the Working Group on ImageGuided Tumor Ablation [28]. Moreover, progression to metastatic disease was documented in $1.9 \%$ (23 of 1234) of the masses in the analysis. A greater number of lesions progressed to metastasis following RFA $(6 / 577,1 \%)$ than following cryoablation $(17 / 657,2.6 \%)$ - a difference that approached statistical significance $(P=0.06)$. Univariate and multivariate regression analyses confirmed that the risk of tumor progression correlated with ablation modality, but not with incidence of malignant pathology nor incidence of unknown pathology in the series that were examined [27]. Although heterogeneous multiinstitutional retrospective data are insufficient to make hard recommendations against a given modality, such as RFA in clinical practice, such findings are provocative and should promote prospective evaluations.

\section{Active surveillance}

Data regarding the natural history of untreated localized renal lesions have been limited [3]. In a recent large series of patients with renal masses under observation, Abouassaly et al. [29••] described 110 elderly patients (median age 81 years, range 76 to 95) with enhancing renal lesions (median size $2.5 \mathrm{~cm}$ ) who enrolled into an active surveillance protocol at the Cleveland Clinic. The strength of this study is the documentation of the Charlson comorbidity index for each patient (median 2, range 0-7) - data that is not available from other active surveillance series. Nearly one third (31\%) of patients expired during the median follow-up time of 24 months (range 1-90) with no deaths being attributed to RCC. The authors do not report whether or not any lesion progressed to metastatic disease, but do document a median renal mass growth rate of $0.26 \mathrm{~cm}$ per year [29・•]. This growth velocity is very similar to the $0.28 \mathrm{~cm}$ per year rate noted in a 2006 meta-analysis of 234 reported lesions [30]. Abouassaly et al. also documented that $43 \%$ of lesions failed to demonstrate growth during the time of follow-up. We have previously reported that between 26 and 33\% of SRMs under surveillance fail to demonstrate any radiographic growth when observed for a median of 29 months. Unfortunately, no clinical predictors of this behavior could be identified in our analysis [31•]. As in the report by Abouassaly et al., [29••] there was no significant correlation of growth rate with lesion size at the start of surveillance [31•]. Similarly, neither patient's age at diagnoses, solid versus cystic appearance, nor the number of imaging studies performed were found to be related to the growth rate. Interestingly, rate of malignancy for masses for which pathological data were available did not differ between lesions that remained stable (83\% malignant) and those that exhibited growth (89\% malignant). Although lack of growth did not correlate with lack of malignancy, no clinically available variable predicted whether a mass would exhibit radiographic growth on surveillance (Table 1Table 1). Nevertheless, none of the zero growth lesions and only one lesion with radiographic growth demonstrated clinical progression. This lesion grew from 2 to $8 \mathrm{~cm}$ over 54 months and progressed to metastatic disease [31•]. Indeed, only 8 examples of progression to metastatic disease are available in the current literature and to our knowledge only one case has been confirmed pathologically. To date, all lesions that progressed to metastatic disease were preceded by rapid radiographic growth (A Kutikov, Kunkle DA, Chen C, et al. Clinical features of small renal masses (SRMs) with documented metastatic progression on active surveillance (AS) protocols. In preparation).

Therefore, a new paradigm may be emerging; namely active surveillance of SRMs with delayed intervention in appropriately selected patients. We recently reviewed our experience with this practice. In 82 patients who underwent at least a 6-month delay (median 14) in intervention for an enhancing renal mass measuring $4 \mathrm{~cm}$ or less (69\% of patients having at least a 12 -month delay), only three were upstaged at resection, and no patient progressed to metastatic disease. 
Estimated 1 and 3-year cancer recurrence-free survival rates were 100 and $99 \%$, respectively [32•]. Reasons for delay were multifactorial and were categorized as absolute (24\%), relative $(21 \%)$, or elective $(55 \%)$. These data suggest that an initial interval of active surveillance is a low-risk approach for management of SRMs. Similarly, Stec et al. [33] recently showed that a surgical delay did not affect patient outcomes in a large cohort of patients ( $n=235$ for patients who had a surgical delay of greater than 1 month). In this study, treatment was delayed more than 6 months for 20 lesions without any apparent deleterious outcomes on cancer-free survival.

\section{Comparison of excision, ablation, and active surveillance}

Given the data, selecting optimal management for the SRM, particularly in the elderly and infirmed has become a clinical dilemma. We recently completed a meta-analysis comparing excision to ablation, and active surveillance [34••]. All published series that reported management of SRMs with open or laparoscopic partial nephrectomy, cryoablation, RFA, or observation were included in the study. Case reports, series that treated patients with hereditary or metastatic RCC were excluded. If redundant data was published, only the most recent report was included. A total of 99 studies encompassing 6471 renal masses comprised the metaanalysis. Data for $5037(77.8 \%)$ distinct masses treated with partial nephrectomy were available in the literature. Outcomes were reported for $496(7.7 \%)$ and $607(9.4 \%)$ masses that were treated with cryoablation and RFA, respectively. A total of 331 (5.1\%) reported masses were managed with active surveillance. Patients treated with partial nephrectomy were statistically significantly younger (60.1 years) than patients treated with ablative technologies (cryo=65.7, RFA=67.2) or active surveillance (68.7). Masses managed with surgery tended to be larger $(3.26 \mathrm{~cm})$ than those treated with cryoablation $(2.56 \mathrm{~cm}, P<0.001)$ or RFA $(2.69, P<0.001)$, but were similar to those treated by active surveillance $(3.04 \mathrm{~cm}, P=0.54)[34 \bullet \bullet]$.

Mean weighted follow-up was longest for the cohort of patients treated with partial nephrectomy (54 months), followed by patients managed with active surveillance (33.3 months). Reported follow-up was shortest for ablation technologies with cryoablation posting a follow-up of 18.3 months and RFA 16.4 months. Differences in follow-up between surgery, active surveillance, and ablation technologies were statistically significant. Local recurrence was reported in $2.6 \%$ of masses treated with partial nephrectomy, $4.6 \%$ of cases treated with cryoablation, and in $11.7 \%$ of masses treated with RFA. Despite shorter follow-up, multivariate analysis revealed a statistically significant higher risk of developing recurrence following cryoablation $(\mathrm{RR}=7.45)$ and $\mathrm{RFA}(\mathrm{RR}=18.23)$ when compared with partial nephrectomy (Table 2 Table 2) [34••]. Importantly, the data confirmed that CSS rates were very high and similar across all modalities.

\section{Conclusion}

Prospective data are limited regarding the optimal management of the SRM. Given the definitive nature of excision and the longest follow-up, surgery remains the standard of care for small and localized RCC. Although cancer specific metrics for ablation appear promising, the impact of these modalities on the natural history of many SRMs is unclear. Available data on ablation technologies suggests that cryoablation is superior to RFA, although this may be a function of the application of the technology rather than the technology itself. Before ablation technologies are adopted as the standard treatment for SRMs in the frail, elderly or comorbid or both, their effect must be measured against the null hypothesis (surveillance). This proof must be sought despite the medical, legal, social and financial disincentives toward active surveillance. Currently, longer follow-up is available for observation than for cryotherapy or RFA with very few patients demonstrating disease progression while on active surveillance. Delayed intervention for SRMs likewise appears to be a safe management strategy. Although patient selection biases are inherent to all retrospective analyses, prospective randomized 
comparisons are both time-consuming and costly. Systematic management of localized RCC based of clinical and pathologic predictors of disease progression must be the long-term goal. Identification of such predictors should be the short-term objective.

\section{Acknowledgments}

This publication was supported in part by grant number P30 CA006927. Additional funds were provided by Fox Chase Cancer Center via institutional support of the Kidney Cancer Keystone Program

\section{References and recommended reading}

Papers of particular interest, published within the annual period of review, have been highlighted as:

- of special interest

•• of outstanding interest

Additional references related to this topic can also be found in the Current World Literature section in this issue (pp. 000-000).

1. Frank FI, Blute FML, Leibovich FBC, et al. Independent validation of the 2002 American Joint Committee on cancer primary tumor classification for renal cell carcinoma using a large, single institution cohort. T J Urol 2005;173:1889.

2. Aron FM, Gill FIS. AT Minimally Invasive Nephron-Sparing Surgery (MINSS) for renal tumours: Part II: Probe ablative therapy. JT European Urology 2007;51:348.

3. Crispen FPL, Uzzo FRG. The natural history of untreated renal masses. BJU Int 2007;99:1203. [PubMed: 17441911]

4. Jemal FA, Siegel FR, Ward FE, et al. Cancer statistics, 2008. CA Cancer J Clin 2008;58:71. [PubMed: 18287387]

5. Jayson FM, Sanders FH. Increased incidence of serendipitously discovered renal cell carcinoma. Urology 1998;51:203. [PubMed: 9495698]

6. Hollingsworth FJM, Miller FDC, Daignault FS, et al. Rising incidence of small renal masses: a need to reassess treatment effect. J Natl Cancer Inst 2006;98:1331. [PubMed: 16985252]

7. Parsons FJK, Schoenberg FMS, Carter FHB. Carter F HB Incidental renal tumors: casting doubt on the efficacy of early intervention. Urology 2001;57:1013. [PubMed: 11377295]

8. Kunkle FDA, Haas FNB, Uzzo FRG. Adjuvant therapy for high-risk renal cell carcinoma patients. Curr Urol Rep 2007;8:19. [PubMed: 17239313]

9. Hafez FKS, Fergany FAF, Novick FAC. Nephron sparing surgery for localized renal cell carcinoma: impact of tumor size on patient survival, tumor recurrence and TNM staging. The Journal of Urology 1999;162:1930. [PubMed: 10569540]

10. Moinzadeh FA, Gill FIS, Finelli FA, et al. Laparoscopic partial nephrectomy: 3 year followup. The Journal of Urology 2006;175:459. [PubMed: 16406971]

11•. Weight FCJ, Fergany FAF, Gunn FPW, et al. The impact of minimally invasive techniques on open partial nephrectomy: a 10-year single institutional experience. The Journal of Urology 2008;180:84. [PubMed: 18485409] This study documents practice pattern shifts at a large tertiary referral center because advent of minimally invasive ablative technology. At the Cleveland Clinic treatment of anatomically amenable SRMs is now performed with minimally invasive ablative techniques, whereas more complex lesions are still treated with partial nephrectomy. Despite this trend, volume of partial nephrectomies performed at this institution has remained stable.

12. Hoffmann FNE, Bischof FJC. The cryobiology of cryosurgical injury. Urology 2002;60:40. [PubMed: 12206847]

13. Campbell FSC, Krishnamurthi FV, Chow FG, et al. Renal cryosurgery: experimental evaluation of treatment parameters. Urology 1998;52:29. [PubMed: 9671865] 
14. Woolley FML, Schulsinger FDA, Durand FDB, et al. Effect of freezing parameters (freeze cycle and thaw process) on tissue destruction following renal cryoablation. J Endourol 2002;16:519. [PubMed: 12396446]

15••. Weight FCJ, Kaouk FJH, Hegarty FNJ, et al. Correlation of radiographic imaging and histopathology following cryoablation and radio frequency ablation for renal tumors. The Journal of Urology 2008;179:1277. [PubMed: 18280507] Excellent report by authors who performed biopsy 6 months following treatment of SRMs with either cryotherapy or RFA. Biopsy results were correlated with posttherapy radiographic findings.

16. Atwell FTD, Farrell FMA, Callstrom FMR, et al. Percutaneous cryoablation of 40 solid renal tumors with US Guidance and CT monitoring: initial experience. Radiology 2007;243:276. [PubMed: 17329689]

17. Bandi FG, Wen FCC, Hedican FSP, et al. Cryoablation of small renal masses: assessment of the outcome at one institution. BJU Int 2007;100:798. [PubMed: 17822460]

18. Finley FDS, Beck FS, Box FG, et al. Percutaneous and laparoscopic cryoablation of small renal masses. The Journal of Urology 2008;180:492. [PubMed: 18550087] Cryoablation can be performed percutaneously or laparoscopically. Objective comparison of percutaneous and laparoscopic cryoablation techniques for treatment of SRMs is reported for a small cohort of patients $(n=37)$ from the Ralph Clayman group.

19•. Bandi FG, Hedican FS, Moon FT, et al. Comparison of postoperative pain, convalescence, and patient satisfaction after laparoscopic and percutaneous ablation of small renal masses. J Endourol 2008;22:963. [PubMed: 18643721] This manuscript compares laparoscopic and percutaneous cryoablation techniques. Despite shorter convalescence for the percutanous approach, patient satisfaction was comparable for both groups.

20. Badwan FK, Maxwell FK, Venkatesh FR, et al. Comparison of laparoscopic and percutaneous cryoablation of renal tumors: a cost analysis. J Endourol 2008;22:1275. [PubMed: 18578660]

21. Klingler FHC. Kidney cancer: energy ablation. Curr Opin Urol 2007;17:322. [PubMed: 17762624]

22. Goldberg FSN, Gazelle FGS, Mueller FPR. Thermal ablation therapy for focal malignancy: a unified approach to underlying principles, techniques, and diagnostic imaging guidance. Am J Roentgenol 2000;174:323. [PubMed: 10658699]

23. Stern FJM, Svatek FR, Park FS, et al. Intermediate comparison of partial nephrectomy and radiofrequency ablation for clinical T1a renal tumours. BJU Int 2007;100:287. [PubMed: 17617136]

24••. Levinson FAW, Su FL-M, Agarwal FD, et al. Long-Term Oncological and Overall Outcomes of Percutaneous Radio Frequency Ablation in High Risk Surgical Patients With a Solitary Small Renal Mass. J Urol 2008;180:499. [PubMed: 18550123] Long-term oncological follow-up for minimally invasive ablative techniques is sparse. This multiinstitutional series reports on 31 patients with longterm follow up (mean 61.6 months, range 41-80) that underwent treatment of SRM with RFA in a solitary kidney.

25. Cadeddu, JA. Re: Correlation of radiographic imaging and histopathology following cryoablation and radio frequency ablation for renal tumors. In: Weight, JW.; Kaouk, JH.; Hegarty, NJ., et al., editors. J Urol. Vol. 179. 2008. p. 1277-1283.J Urol 2008;179:1281.

26••. Raman FJD, Stern FJM, Zeltser FI, et al. Absence of viable renal carcinoma in biopsies performed more than 1 year following radio frequency ablation confirms reliability of axial imaging. J Urol 2008;179:2142. [PubMed: 18423723] Important report suggesting that presence of tumor architecture on early ( $<12$ months) biopsy following RFA may be unreliable. The authors document that no viable tumor was presents in lesions $(n=20)$ that was biopsied for more than 1 year following RFA.

27. Kunkle DA, Egleston BL, Uzzo RG. Cryoablation versus radiofrequency ablation for the treatment of small renal masses. Cancer. in press

28. Matin FSF, Ahrar FK, Cadeddu FJA, et al. Residual and recurrent disease following renal energy ablative therapy: a multiinstitutional study. J Urol 2006;176:1973. [PubMed: 17070224]

29••. Abouassaly FR, Lane FBR, Novick FAC. Active surveillance of renal masses in elderly patients. J Urol 2008;180:505. [PubMed: 18550113] Largest active surveillance series of SRMs to date $(n=110)$. All patients had Charlson comorbidity index documented at diagnosis. 
30. Chawla FSN, Crispen FPL, Hanlon FAL, et al. The natural history of observed enhancing renal masses: meta-analysis and review of the world literature. J Urol 2006;175:425. [PubMed: 16406965]

31•. Kunkle FDA, Crispen FPL, Chen FDY, et al. Enhancing renal masses with zero net growth during active surveillance. J Urol 2007;177:849. [PubMed: 17296355] Some lesions may not exhibit growth while on observation. This is the first study examining the phenomenon of zero net growth of lesions on active surveillance.

32•. Crispen FPL, Viterbo FR, Fox FEB, et al. Delayed intervention of sporadic renal masses undergoing active surveillance. Cancer 2008;112:1051. [PubMed: 18286513] Delayed intervention may be necessary or desirable in a subset of patients with SRMs. This study examines the safety of this approach.

33. Stec FAA, Coons FBJ, Chang FSS, et al. Waiting time from initial urological consultation to nephrectomy for renal cell carcinoma--does it affect survival? J Urol 2008;179:2152. [PubMed: 18423724]

34••. Kunkle FDA, Egleston FBL, Uzzo FRG. Excise ablate or observe: the small renal mass dilemma-a meta-analysis and review. J Urol 2008;179:1227. [PubMed: 18280512] Decision between surgery, ablation, or active surveillance is often qualitative. Here, authors perform a meta-analysis of the world literature and attempt to quantify effectiveness of each approach for treatment of SRMs. 
Table 1

Comparison of characteristics between lesions that grew and failed to grow on active surveillance

\begin{tabular}{llll}
\hline Characteristic & Zero growth lesions & $\begin{array}{l}\text { Lesions that grew on active } \\
\text { surveillance }\end{array}$ & -value \\
\hline Number $(\%)$ & & $71(67)$ & - \\
Median pt age & $35(33)$ & 73 & 0.96 \\
Median initial size $(\mathrm{cm})$ & 71 & 2 & 0.5 \\
Median follow-up (mo) & 2 & 30 & 0.066 \\
Median growth (cm/yr) & 25 & 0.31 & $<0.00001$ \\
Median imaging studies & 0 & 6 & 0.76 \\
No. cystic lesions $(\%)$ & 5 & $9(13)$ & 1 \\
No. malignant pathology/total no. $(\%)$ & $4(11)$ & $32 / 36(89)$ & 0.56 \\
No. metastatic progression $(\%)$ & $5 / 6(83 \%)$ & 1 & 1 \\
\end{tabular}

Adapted from [31•]. 
Table 2

Relative risk of local recurrence or metastatic disease as derived from a meta-analysis of available literature

\begin{tabular}{|c|c|c|c|}
\hline SRM management strategy & $\mathbf{n}$ & Relative risk of local recurrence & $\begin{array}{l}\text { Relative risk of metastatic } \\
\text { progression }\end{array}$ \\
\hline $\begin{array}{l}\text { Partial nephrectomy } \\
\text { Cryoablation } \\
\text { RFA } \\
\text { Active surveillance }\end{array}$ & $\begin{array}{l}5037 \\
496 \\
607 \\
331\end{array}$ & $\begin{array}{l}1.00 \\
7.45 \\
18.23 \\
-\end{array}$ & $\begin{array}{l}1.00 \\
1.24 \\
3.21 \\
0.11\end{array}$ \\
\hline
\end{tabular}

Local recurrence was defined as radiographic or pathological evidence of residual disease or recurrent disease (adjacent to previous tumor bed in the case of partial nephrectomy) regardless of time to recurrence. Adapted from [34••]. 